\title{
Wolbachia is not all about sex: male-feminizing Wolbachia alters the leafhopper Zyginidia pullula transcriptome in a mainly sex-independent manner
}

\author{
Hosseinali Asgharian $^{1 *}$, Peter L. Chang ${ }^{1}$, Peter J. Mazzoglio ${ }^{2}$ and llaria Negri ${ }^{2}$ \\ ${ }_{1}^{1}$ Program in Molecular and Computational Biology, Department of Biological Sciences, University of Southern California, Los Angeles, CA, USA \\ ${ }^{2}$ DISAFA - Department of Agricultural, Forest and Food Sciences, University of Torino, Grugliasco (TO), Italy
}

Edited by:

M Pilar Francino, Center for Public Health Research, Spain

Reviewed by:

Anna Carolin Frank, University of

California Merced, USA

Natacha Kremer, Université Claude

Bernard Lyon 1, France

\section{${ }^{*}$ Correspondence:}

Hosseinali Asgharian, Program in Molecular and Computational

Biology, Department of Biological

Sciences, University of Southern California, RRI 413M, 1050 Childs

Way, Los Angeles, CA 90007, USA

e-mail: asgharia@usc.edu
Wolbachia causes the feminization of chromosomally male embryos in several species of crustaceans and insects, including the leafhopper Zyginidia pullula. In contrast to the relatively well-established ecological aspects of male feminization (e.g., sex ratio distortion and its consequences), the underlying molecular mechanisms remain understudied and unclear. We embarked on an exploratory study to investigate the extent and nature of Wolbachia's effect on gene expression pattern in Z. pullula. We sequenced whole transcriptomes from Wolbachia-infected and uninfected adults. 18147 loci were assembled de novo, including homologs of several Drosophila sex determination genes. A number of transcripts were flagged as candidate Wolbachia sequences. Despite the resemblance of Wolbachia-infected chromosomal males to uninfected and infected chromosomal females in terms of sexual morphology and behavior, principal component analysis revealed that gene expression patterns did not follow these sexual phenotype categories. The principal components generated by differentially expressed genes specified a strong sex-independent Wolbachia effect, followed by a weaker Wolbachia-sexual karyotype interaction effect. Approaches to further examine the molecular mechanism of Wolbachia-host interactions have been suggested based on the presented findings.

Keywords: Wolbachia infection, male feminization, principal component analysis (PCA), Zyginidia pullula transcriptome, transcriptome de novo assembly, host-symbiont interactions

\section{INTRODUCTION}

Wolbachia is an intracellular symbiont alpha-proteobacterium that infects a wide range of arthropods and nematodes (Schulenburg et al., 2000; Werren et al., 2008). It is often transmitted vertically from females through the eggs to their future progeny; although, horizontal transfer between hosts has also been documented (Werren et al., 1995; Cordaux et al., 2001). Studying the mechanism of Wolbachia-host interactions is fascinating for many reasons. Wolbachia is capable of inducing several intriguing sex-related phenotypes in its hosts, including male killing (MK), in which infected males die during embryonic or larval stages; male feminization (MF), that is the development of genetic males into females; thelytokous parthenogenesis (TP) in which infected virgin females produce daughters. All of these phenotypes distort the progeny sex ratio in favor of females thus ensuring higher transmission rate of Wolbachia to the next generation of hosts (Werren et al., 2008; White et al., 2013). Another fascinating effect of the infection is cytoplasmic incompatibility between gametes (CI), which results in aberrant or considerably reduced offspring production, if uninfected females mate with infected males, or if the parents are infected with different Wolbachia strains (Werren et al., 2008; White et al., 2013). In this case, infected females possess a reproductive advantage compared to uninfected ones, and this again ensures the spreading of
Wolbachia into the host population. Fast transition between the four phenotypes in the course of the coevolution of Wolbachia and its hosts hints that similar molecular mechanisms might underlie the apparently different effects (Ma et al., 2014). Due to its enormous host range, Wolbachia may have played a crucial role in the evolution of sex determination system and reproductive strategies in arthropods (Cordaux et al., 2011; Awrahman et al., 2014; Ma et al., 2014).

Various approaches have been employed to investigate the Wolbachia-host interactions in naturally infected and uninfected strains (Hoffmann et al., 1990; Negri et al., 2006; Riparbelli et al., 2012), experimentally inoculated cell lines (Noda et al., 2002; Xi et al., 2008), and antibiotic treated specimens (Hoffmann et al., 1990; Casiraghi et al., 2002). Although Wolbachia is an obligate intracellular symbiont natuarally, protocols have been developed to keep it viable in cell-free media for days; however, no replication occurs in the extracellular phase (Rasgon et al., 2006; Gamston and Rasgon, 2007). The experimental/analytical techniques comprised a wide range including classical crossing and fecundity measurements (e.g., Hoffmann et al., 1990; Dunn et al., 2006), microscopic approaches (in situ hybridizations, electron microscope and immunohistochemical techniques for bacterium detection inside hosts and cells, tissues, etc.) (e.g., Negri et al., 2008; Fischer et al., 2011), gene expression analysis (e.g., Xi et al., 
2008; Kremer et al., 2009, 2012; Hughes et al., 2011; Chevalier et al., 2012; Darby et al., 2012; Liu et al., 2014), bioinformatic genome sequence annotation and functional prediction (e.g., $\mathrm{Wu}$ et al., 2004; Foster et al., 2005; Klasson et al., 2008), and mathematical modeling of the ecological consequences of CI or sex ratio distortion (e.g., Taylor, 1990; Turelli, 1994). Despite all these efforts, a coherent mechanistic story of Wolbachia's effect is still lacking. The picture is incomplete even for CI which occurs in Drosophila and is the most extensively studied Wolbachiainduced phenotype; although, cytoskeleton reorganization and asynchrony in nuclear envelope break down and chromosomal condensation of male and female pronuclei after fertilization have been implicated in the process (Serbus et al., 2008; Werren et al., 2008). The other three phenomena are less well understood. TP seems to result from induction of diploidy in species with a haplodiploid sex determination system by production and development of diploid eggs; that is achieved by altering meiosis to produce diploid gametes (Weeks and Breeuwer, 2001), the abortion of the first mitotic division after chromosomal duplication (Pannebakker et al., 2004), or the fusion of the two haploid nuclei after first mitosis of induced eggs (Gottlieb et al., 2002). The molecular bases of MK and MF are least understood but they are suspected to share certain components as $\mathrm{MK}$ is often the result of a lethal and incomplete attempt at feminization of genetic male embryos (Werren et al., 2008). The most direct mechanistic evidence comes from the study of male killing Wolbachia in the moth Ostrinia scapulalis showing that it overrides the karyotypic signal in genetic males to produce the female $d s x$ isoform (Sugimoto and Ishikawa, 2012). This suggests that Wolbachia impacts the sex determination pathway at or above $d s x$. Apart from this direct effect on the pivotal sex determining gene $d s x$, MK or MF Wolbachia infection is reported to be accompanied with defective chromatin remodeling (Riparbelli et al., 2012), induction of host immune response (Chevalier et al., 2012), and epigenetic reprogramming of the host (Negri et al., 2009a).

Zyginidia pullula is a leafhopper with XX/XO male heterogametic sex determination system in which Wolbachia causes feminization of chromosomal males (Negri et al., 2006). Infected female leafhoppers are morphologically indistinguishable from uninfected females; but feminized chromosomal males have an intersex phenotype i.e., they have the upper pygofer appendages, a typical male secondary sexual feature. These appendages show varying degrees of development, from being fully developed in some specimens to being a barely recognizable stump in others (Negri et al., 2006). Feminized males with upper pygofer appendages reduced to a stump have ovaries morphologically similar to uninfected females, whereas those with prominent appendages possess malformed and probably less functional ovaries (Negri et al., 2008). The "degree of feminization" has been shown to be correlated with Wolbachia density in the host tissues in several systems (Jaenike, 2009). We have previously reported that Wolbachia instigates epigenetic reprogramming of Z. pullula (Negri et al., 2009a,b) and probably interacts with the insect hormone biosynthesis pathway to stimulate the production of feminizing hormones (Negri et al., 2010; Negri, 2012). In this study, whole transcriptomes of male and female Zygindia samples (Wolbachia-infected and uninfected) were analyzed with Illumina deep sequencing technique, in order to understand the scope and nature of the Wolbachia-induced change in the host gene expression profile. Our initial idea was that if male feminization is the main consequence of Wolbachia infection, transcriptomes from the three female types (uninfected females, infected females and feminized males) should resemble each other and be different from the only phenotypically male group (uninfected males). In fact, we decided to test the hypothesis that sex reversal is Wolbachia's main effect at the transcriptome level. Were this confirmed, we would proceed to identify differentially expressed genes between the two sexual phenotype groups.

\section{METHODS}

\section{ZYGINIDIA SPECIMENS}

34 overwintering females of $Z$. pullula were collected in the same grass field in north Italy; and were reared individually in the laboratory as described in Negri et al. (2006). Overwintering females have often mated with several males (rarely with only one). By carefully examining the progeny, Wolbachia-infected (i.e., all female brood) and uninfected (i.e., male and female brood) lines were identified. Wolbachia infection was then confirmed by PCR on the mothers and randomly chosen samples from the brood as described in Negri et al. (2006). Morphological investigation as to the presence or absence of upper pygofer appendages lead us to separate feminized males from genetic females in the allfemale (i.e., Wolbachia-infected) lines, and males and females in the uninfected lines. Males from uninfected lines were mated to the physiologically female progeny of the infected lines (consisting of genetic females and males) at each generation to produce the next generation of infected females (and feminized males). This backcrossing to uninfected males was done for at least three generations in the lab. Fifty adults from each of the four different categories of uninfected females (F), uninfected males (M), infected females (FW) and feminized (infected) males (MW) were pooled together for RNA sequencing.

\section{cDNA LIBRARY PREPARATION AND SHORT-READ SEQUENCING}

CDNA libraries were made from male and female specimens of infected and uninfected leafhopper lines. Infected males are phenotypically intersex and exhibit different degrees of feminization depending on the concentration of Wolbachia, ranging from individuals with functional ovaries to individuals with female secondary sexual characters, but possessing testes. We used thoroughly feminized infected males for RNA extraction. RNA purification, cDNA synthesis and Illumina library construction were performed using the protocols of Mortazavi et al. (2008), with the following modifications: total RNA, mRNA and DNA were quantified using a Qubit fluorometer (Invitrogen); mRNA fragmentation was performed using Fragmentation Reagent (Ambion) for a $3 \mathrm{~min}$ and $50 \mathrm{~s}$ incubation at $70^{\circ} \mathrm{C}$ and subsequently cleaned through an RNA cleanup kit (Zymo Research); additional DNA and gel purification steps were conducted using Clean and Concentrator kits (Zymo Research). Each sample library was sequenced as pair-ended 76-base reads on an Illumina Genome Analyzer II. 


\section{DE NOVO TRANSCRIPTOME ASSEMBLY AND EXPRESSION LEVEL CALCULATION}

Due to the sensitive nature of de novo assembly, it is critical that the reads used to generate contigs have the highest sequencing quality. Reads were removed from consideration in the de novo assembly if they had a terminal phred (Ewing and Green, 1998) quality value less than 15, or contained more than 2 unknown nucleotides (i.e., $N$ ). Reads were also filtered due to similarities to known PCR primer and Illumina Adapter sequences. Using the reads pooled from all of the four samples that were not filtered out, the de novo assembly program Velvet (version 1.0.15) (Zerbino and Birney, 2008) was used in conjunction with a custom post-processing algorithm capable of retaining information from alternative splices (Sze et al., 2012) to assemble short reads into contigs, using sequence overlap information until the contigs could no longer be extended. Velvet was run under the following settings with a kmer length of 35: -cov_cutoff auto -max_branch_length 0 -max_divergence 0 -max_gap_count 0 read_trkg yes. Sequenced reads that were kept as pairs and not filtered out together or separately were treated as "-shortPaired" with insert length of 175 bases and standard deviation of 75 bases. Single end reads that were not filtered out were treated as "-short."

With the set of de novo assembled sequences serving as a reference, reads from each of the individual samples were mapped using the Burrows-Wheeler Aligner (BWA) (Li and Durbin, 2009). The number of reads that mapped to the contigs of each gene was tabulated and normalized to calculate FPKM (Fragments Per Kilobase Of Exon Per Million Fragments Mapped). Additional normalization among all samples was performed using the TMM protocol (Trimmed Mean of M-values) outlined in Robinson and Oshlack (2010), which takes into account differences in overall RNA populations across samples and is one of several methods used to evaluate RNA sequencing data. Normalization was implemented using the edgeR package in R (Robinson et al., 2010). All statistical analyses and graphs evaluating consistency between samples were produced using R v2.13.0 (R Development Core Team, 2011).

\section{GENE FUNCTIONAL ANNOTATION AND CLASSIFICATION}

Blast2GO v.2 (Götz et al., 2008) and WEGO (Ye et al., 2006) were used to obtain Gene Ontology (GO) annotations. Genes were also annotated using a BLASTX search (Altschul et al., 1990) (Expected value $<1.00 \mathrm{e}-05$ ) to the $\mathrm{nr}$ protein database available from GenBank as well as to the set of protein sequences available from the Drosophila melanogaster 5.34 and the pea aphid Acyrthosiphon pisum 2.1 releases. We chose the annotation with the highest BLAST score as long as the span of the alignment was greater than $80 \%$ of the length of the contig under query. For genes that did not report any hits, we lowered the minimum span to $40 \%$ of the length, choosing the annotation with the highest BLAST score having Expected value $<1.00 \mathrm{e}-05$.

\section{PRINCIPAL COMPONENT ANALYSIS OF GENE EXPRESSION VALUES}

Expression values were cleaned of extreme outliers, quartilenormalized and log-transformed before they were used for PCA. To make sure the result were not artifacts of the data preparation method, PCA was repeated on the raw (not normalized, not log-transformed) expression values as well as after several different outlier-filtering and normalization strategies. These statistical procedures were done in SAS 9.3.

\section{RESULTS}

\section{SHORT-READ SEOUENCING AND DE NOVO ASSEMBLY}

The mRNA population was analyzed with Illumina deep sequencing of male and female Zygindia samples with and without Wolbachia infection. The pooled data from all samples had a total of $50 \mathrm{M}$ pair-ended reads that were 76 bases long. All Illumina sequences are available for download at the NCBI Short Read Archive under the BioProject PRJNA171390. After sequences were filtered based on quality and matches to adapter and primer sequences, the $38 \mathrm{M}$ reads from all four samples were pooled together and run through Velvet and the post-processing algorithm. Eventually, 18,147 loci and a total of 27,236 transcripts were assembled; multiple transcripts of a locus pertained often to different splicing isoforms and occasionally to largely differentiated alleles. The transcripts ranged in lengths from $291 \mathrm{bp}$ to $15,389 \mathrm{bp}$, with mean and median lengths of $1006 \mathrm{bp}$ and 702 bp, respectively. This assembly included a fairly large number of long transcripts: $25 \%$ were longer than $1250 \mathrm{bp}$ and $10 \%$ were longer than 2000 bp. Of the 18,147 loci, 14,068 (77.5\%) had a single isoform and the remaining $22.5 \%$ had multiple ones. Transcripts within a locus were subsequently collapsed into a single "representative locus sequence" by using ClustalW to run a multiple sequence alignment and identifying the locus consensus sequence. Mean and median lengths of consensus sequences were $900 \mathrm{bp}$ and $618 \mathrm{bp}$, respectively. The total length of all loci consensus sequences was $16.3 \mathrm{Mb}$.

\section{GENE FUNCTIONAL ANNOTATION AND CLASSIFICATION}

6946 loci, corresponding to $38 \%$ of the entire dataset, were Gene Ontology annotated with Blast2GO. The consensus sequences were also aligned using a BLASTX search to the $\mathrm{nr}$ protein database available from GenBank as well as to the set of protein sequences available from Flybase and the aphid genome. Table 1 shows the proportion of cases that resulted in a hit where the length of the alignment was greater than $80 \%$ or $40 \%$ of the length of the query (leafhopper sequence). One might very crudely attribute the $80 \%$ alignment span hits to true genic homology and the $40 \%$ alignment span hits to conserved domains.

Table 1 | Summary statistics of Zyginidia transcripts homology search.

\begin{tabular}{lcc}
\hline & $\mathbf{4 0} \%$ homology length & $\mathbf{8 0} \%$ homology length \\
\hline Genbank & $60 \%$ & $39 \%$ \\
Flybase & $48 \%$ & $26 \%$ \\
Pea aphid & $81 \%$ & $32 \%$ \\
\hline
\end{tabular}

$40 \%$ homology length indicates that the length of the homologous segment covers at least $40 \%$ of the query (leafhopper) sequence. A correspondingly similar definition applies to the $80 \%$ homology length category. The percent values in the table cells show what percent out of all loci (18147) fit each criterion when Blasted against the designated dataset. 
A number of genes potentially involved in the leafhopper sex determination were identified through homology search with the Drosophila sex determination genes. Although pea aphid is Zyginidia's closest relative with a reference genome sequence (The International Aphid Genomics Consortium, 2010), the functional annotation for this genome is not as complete as that of Drosophila. Sex determining genes of pea aphid have been found based on homology with Drosophila sequences and lack direct experimental verification (The International Aphid Genomics Consortium, 2010). Therefore, we decided to use Drosophila sequences as the reference set. Figure 1 depicts the canonical sex determination pathway in Drosophila. Homologs of several Drosophila sex determination genes were identified among the transcripts including $d s x$ (doublesex), tra-2 (transformer-2), vir (virilizer), fl(2)d (female lethal $d$ ), snf (sans fille) and ix (intersex). No leafhopper homologs could be identified for tra (transformer), sxl (sex lethal), fru (fruitless) or her (hermaphrodite). Table 2 shows the expression levels for the identified leafhopper sex determination genes.

Seventeen genes in our dataset were flagged as likely Wolbachia sequences according to the Blast results against the NCBI dataset. Bacterial origin seems very probable for a number of these transcripts based on the expression levels in infected and uninfected lines, plus high similarity to known Wolbachia sequences (Table 3). These sequences were Blasted against the aphid genome to check if there was an indication of horizontal transfer; they were also Blasted against the Drosophila genome as a distant outgroup (Table 3 ).

\section{PRINCIPAL COMPONENT ANALYSIS OF TRANSCRIPTOMES}

Principal component analysis on transcriptomes of the four leafhopper samples surprisingly revealed that Wolbachia infection changes the host transcriptome extensively and the effect is by no means limited to sex-reversal. As evident in Figure 2,

Table 2 | Homologs of Drosophila sex determination genes in the Zyginidia transcriptome and their normalized expression levels.

\begin{tabular}{llcrrrr}
\hline Locus & Fly homolog & $\begin{array}{c}\text { Multiple } \\
\text { isoforms? }\end{array}$ & FW & MW & F & M \\
\hline 5652 & dsx & Y & 11.6 & 49.4 & 11.7 & 19.7 \\
8743 & tra-2 & Y & 5.9 & 13.2 & 8.8 & 26.1 \\
5015 & vir & N & 20.2 & 9.9 & 16.1 & 23.2 \\
10229 & fl(2)d & N & 17.4 & 32.9 & 40.9 & 63.3 \\
21060 & snf & Y & 0 & 3.3 & 20.5 & 10.9 \\
18743 & ix & N & 5.9 & 11.6 & 81.8 & 43.9
\end{tabular}

$F W$, infected female; $M W$, infected feminized male (intersex female); $F$, uninfected female; $M$, uninfected male.

\section{Femaleness}

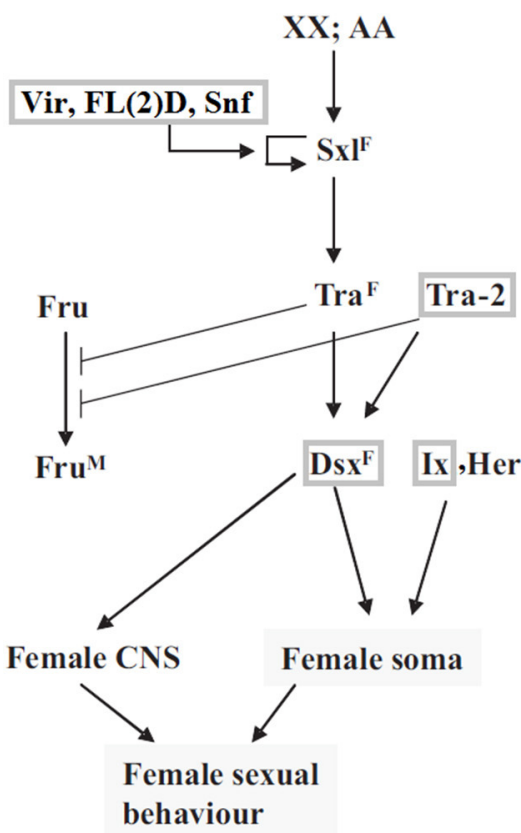

FIGURE 1 | Sex determination pathway in Drosophila, modified from Sánchez (2008). Sxl ${ }^{F}$ and $\left.S x\right|^{M}$ refer to functional female and nonfunctional male isoforms of the Sxl protein, respectively. $\mathrm{Tra}^{\mathrm{F}}$ is the functional female form of the Transformer protein, which in conjunction with the constitutive gene product Tra-2 controls female-specific splicing of dsx and fru. snf, vir and $F L(2) D$ are required for late female-specific splicing of $S x l$ but play no part

\section{Maleness}

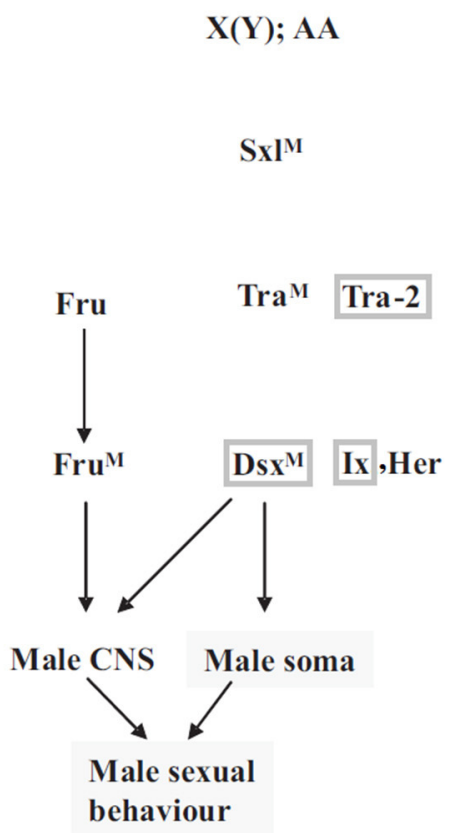

in determining early Sxl splicing pattern. The genes for which Z. pullula homologs have been identified in this study, are boxed in gray. For more details on the regulation and function of these genes, refer to (Sánchez, 2008; Gempe and Beye, 2011) or other similar resources. Reproduced with permission from The International Journal of Developmental Biology (Int. J. Dev. Biol.) (2008) Vol:52, pp. 837-856. 


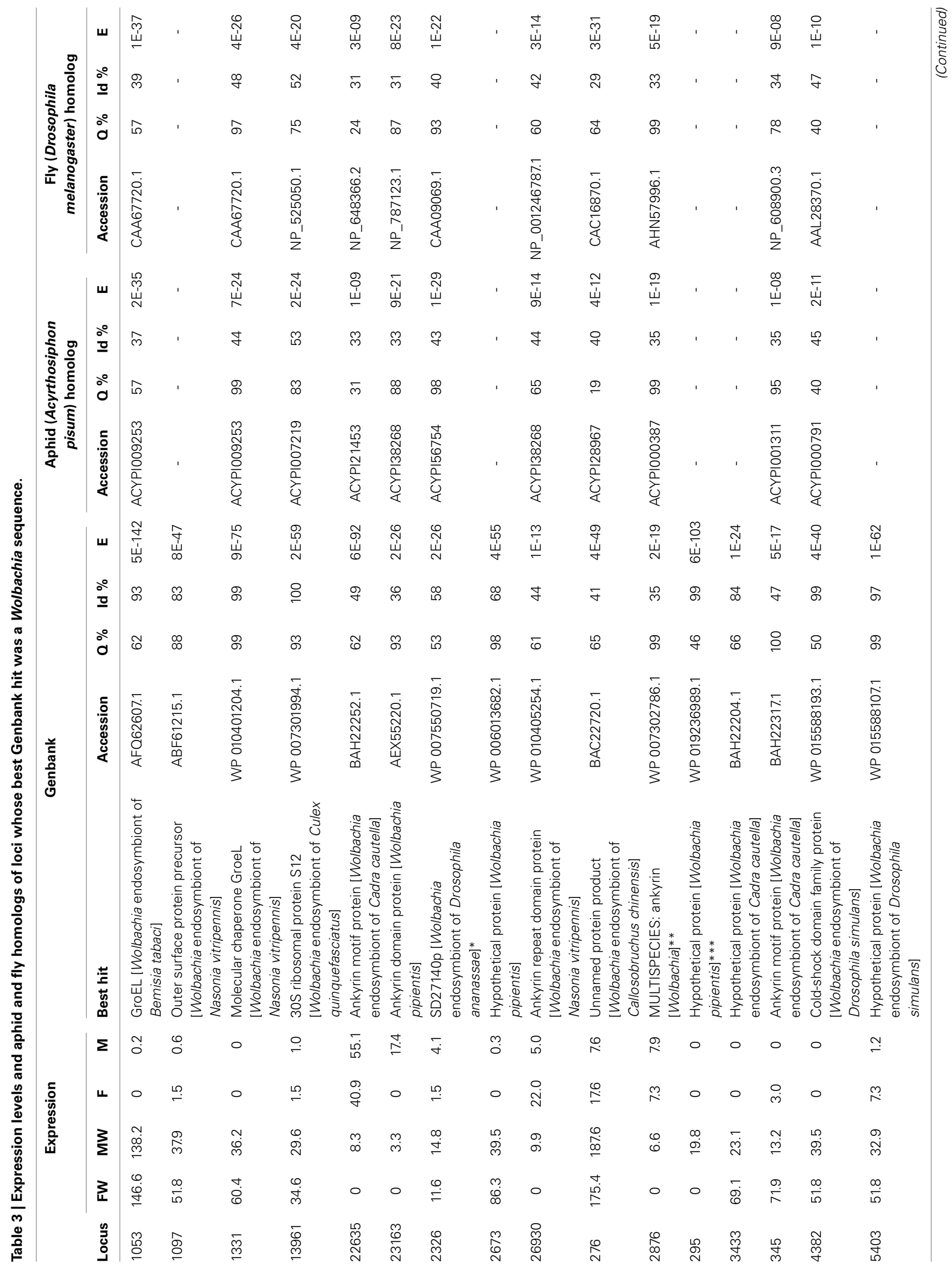




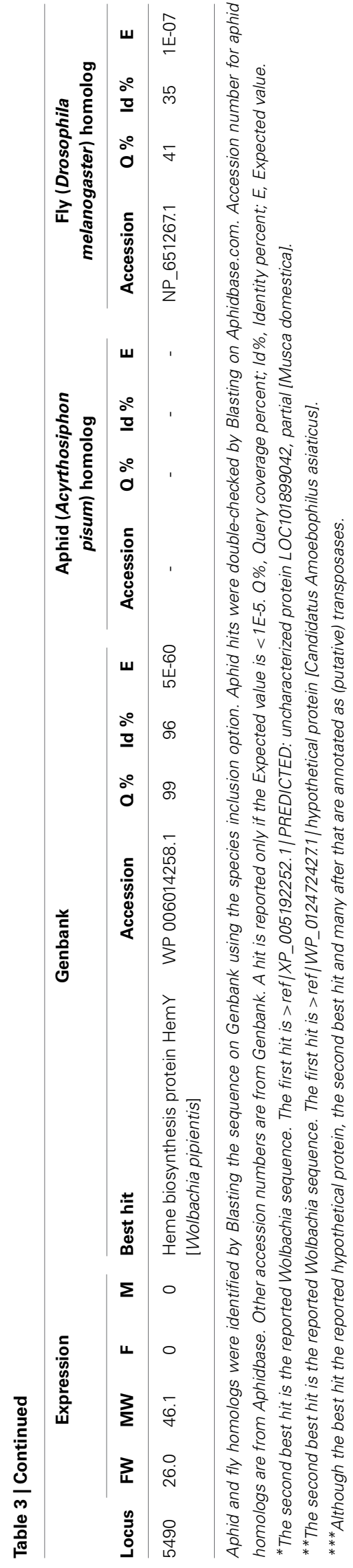

the first PC (explaining $66.46 \%$ of variance) is highly correlated with all of the samples indicating that the expression of most genes is not significantly altered by Wolbachia and is similar across all samples. By the second PC (explaining 20.36\% of variance), Wolbachia infected male and female samples cluster together and uninfected male and female cluster together. This PC is generated by genes whose expression is changed by Wolbachia consistently regardless of sexual karyotype or phenotype. The third PC (explaining $7.97 \%$ of variance) indicates an interaction term: $\mathrm{F}$ and $\mathrm{M}$ are similar and stand in the middle of the scale, with MW and FW occupying the opposite sides of them. This PC is generated by genes that are expressed similarly in uninfected males and females, and Wolbachia infection changes their expression in opposite ways in chromosomal males and females. Overall, sex inversion does not seem to be the only or even the biggest effect of Wolbachia on gene expression patterns in Zyginidia, even if it is the most conspicuous phenotypic consequence; otherwise, we would expect the three phenotypically female groups (F, FW and $\mathrm{MW}$ ) to cluster together and the only male group (M) to stand separate from them. None of the PCs show such a pattern. PCA was repeated on expression values without the initial outlier filtering, and applying several different normalization and transformation strategies; they all yielded the same picture as described above: the main effect was invariably the presence or absence of Wolbachia regardless of sex (details not shown).

\section{DISCUSSION}

We assembled the $Z$. pullula transcriptome de novo and produced 18,147 loci and 27,236 transcripts with a total consensus sequence length of $16.3 \mathrm{Mb}$. These numbers were well within the expected range based on the aphid genome information. The aphid genome was reported to contain 11,089 highly supported RefSeq gene models with a total exonic length of $21.6 \mathrm{Mb}$; adding the gene models from six other gene prediction programs, a total of 34,604 non-redundant gene models with the total exonic length of $35.7 \mathrm{Mb}$ were described (The International Aphid Genomics Consortium, 2010). The true number of genes is purportedly a number between those two estimates. Hence, our de novo assembly of the transcriptome seems to have captured a reasonable proportion of the expressed genes.

The results of sequence homology search (Table 1) confirm the closer relatedness of $Z$. pullula to A. pisum (the aphid) than to Drosophila. A caveat to this analysis is the extensive set of duplications in the aphid genome (The International Aphid Genomics Consortium, 2010). Without a leafhopper reference genome, we do not know if the same wave of duplications has affected $Z$. pullula or not; however, there was an indication in our data that it might have. By visual inspection of the sequences that were annotated as isoforms of a single locus computationally, we realized that some of them did not show signatures of known alternative splicing patterns; but looked like highly differentiated alleles (details not shown). These may indeed be paralogous sequences in the process of divergence. Further investigation, including the sequencing of single individuals rather than pools of them, will be required to separate paralogy from allelic variation.

A number of leafhopper sex determination genes were identified based on homology with fly sequences (Table 2). 


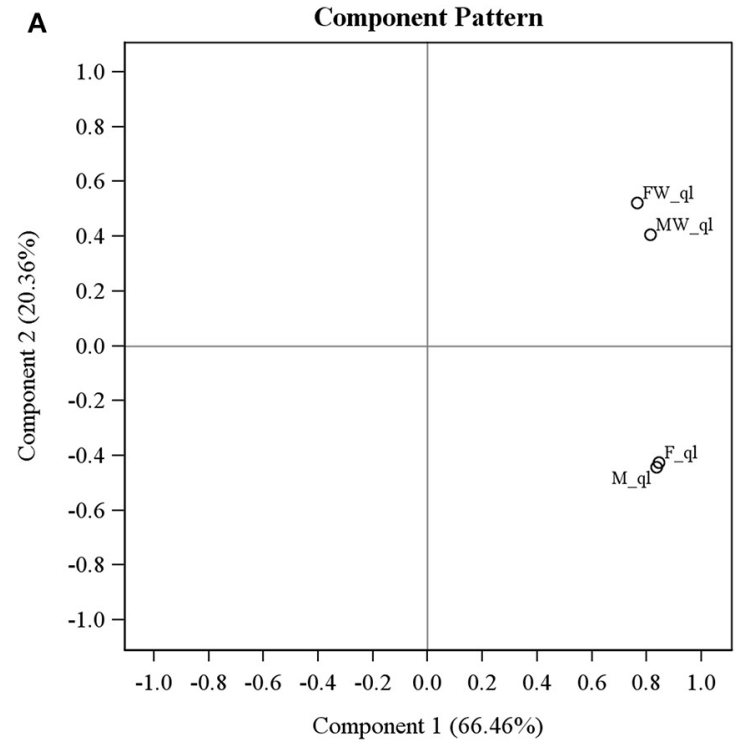

FIGURE 2 | Principal component analysis of gene expression levels in four Zyginidia samples: infected females (FW), infected males or intersex females (MW), uninfected females (F) and uninfected males (M).

The first three PCs are depicted here. PC1 is correlated with all sample labels

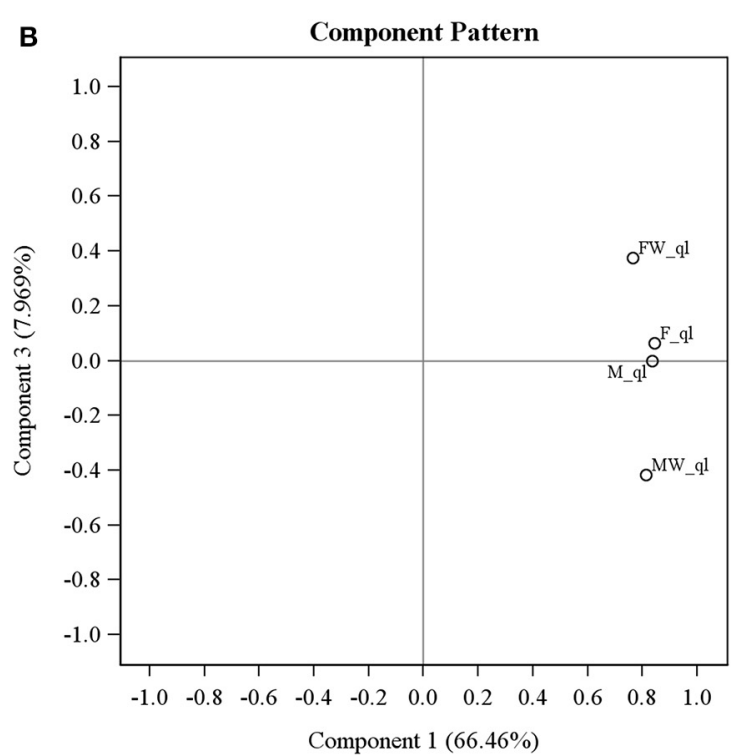

but PC2 separates the infected and uninfected samples conclusively. PC3 reveals an interaction term between Wolbachia status and sexual karyotype. The "_ql" suffix after line names means that the expression values were quantile-normalized and log transformed. (A) PC1 vs. PC2; (B) PC1 vs. PC3.
Insect sex determination machinery has evolved around the transformer-doublesex axis (Sánchez, 2008); tra is the fast evolving component responsible for receiving the signal-sometimes through mediators- from the upstream sex determining factors (chromosomal constituent, incubation temperature, etc.), and $d s x$ is the conserved switch that relays this signal down to the developmental processes (Sánchez, 2008; Verhulst et al., 2010). It is, therefore, not surprising that we found a homolog for $d s x$ and not for tra in our dataset. The short length of the aligned segments prevented reliable assignment of male and female isoforms; but these initial results can be used to design primers to extract the whole genes from the leafhopper genome. Future experiments can then follow the flow of the signal in the sex determination pathway to identify where the cascade is diverted to female development in Wolbachia-infected genetic males. In the moth O. scapulalis, the impact point is somewhere above the level of $d s x$ (Sugimoto and Ishikawa, 2012). Having the sequences of $d s x$ male and female isoforms, one could check whether this is also true in leafhoppers. Unfortunately, the lack of replicates in our preliminary data makes it impossible to assess the significance of differential expression of genes across our four groups (FW, MW, $\mathrm{F}$, and $\mathrm{M}$ ). This is another task that remains to be done in future projects. In addition, development of X-linked sequence markers will enable early sexing of the embryos (based on the female XX / male XO karyotypes) through quantitative PCR; and facilitate the study of early developmental processes in infected and uninfected specimens.

We found a number of Wolbachia-related transcripts in the sequenced cDNA libraries (Table 3). The loci expressed mainly in infected lines with great similarity to known Wolbachia sequences are likely to have Wolbachia origin (e.g., loci 1053, 1097, 1331, and 13961). Curiously, a couple of loci are expressed primarily in the uninfected lines (e.g., locus 22635). At this point, we do not have a hypothesis as to the reason behind this observation. Repeating the experiments with replicates and higher sequencing depth would be the first step to confirm the reproducibility of these patterns. Our protocol of mRNA purification for creation of cDNA libraries involved a hybridization step with oligo-T ligands, which targets the eukaryotic mRNA poly-A tails; therefore, it will be necessary to employ a different purification strategy in order to capture most of the poly-A lacking bacterial mRNAs. Table 3 shows that several of the Wolbachiarelated sequences code for Ankyrin-repeat proteins. Wolbachia genomes are well known for containing an extraordinarily high number of these genes (Wu et al., 2004; Iturbe-Ormaetxe et al., 2005). Gene transfer between Wolbachia and mosquito hosts has been previously reported (Woolfit et al., 2009). PCR experiments and phylogenetic analyses have confirmed horizontal gene transfer from bacterial endosymbionts to the aphid genome (The International Aphid Genomics Consortium, 2010). Similar approaches will be required to confirm bacterial or insect origin for the transcripts listed in Table 3. We tried to check for possible aphid lineage-specific horizontal transfers by asking whether a likely Wolbachia transcript shows high sequence similarity to an aphid sequence, but not a fly sequence; none of the loci in Table 3 expressed such a pattern. One of the Wolbachia-related transcripts showed a degree of homology with the aphid vasa gene (locus 4382). Almost identical homologs of this sequence exist in the three published Wolbachia genomes (Blast results not shown); its homologs in fly, leafhopper and the published Wolbachia genomes are characterized or predicted ATP-dependent RNA helicases. vasa has been implicated in transmission of maternal effects and sex determination in clams (Milani et al., 2011). It will be very interesting to check if products 
of host-homologous genes are actually exported out by Wolbachia into the host cell.

We used natural isolates of infected and uninfected leafhoppers for our comparisons with no antibiotic treatment. This relieved our comparisons from the confounding effects of antibiotic treatments on the host physiology. The rationale behind the traditional use of antibiotics to cure the infected lines from Wolbachia is to obtain infected and uninfected lines with the same genetic background. However, antibiotics can change the host physiology substantially, and quite remarkably, their effect can perpetuate through several generations of unexposed progeny (Ballard and Melvin, 2007; Zeh et al., 2012; Fridmann-Sirkis et al., 2014). We avoided the use of antibiotics completely and achieved homogenous genetic backgrounds among samples by taking advantage of repeated backcrossing of infected females to uninfected males. We collected all of our founder specimens from the same leafhopper population in a grass field. In the sampled population, the sex-ratio was only moderately female biased, with a moderate prevalence of the infection ( $\sim 1: 1.8$ male:female, Wolbachia infection rate $\sim 30 \%$ of the collected females; Negri I., unpublished data). As uninfected males are the only physiological males in existence, all the "egg-laying females" (in the field and in the lab, including the females used in this study) always mate with (and only with) uninfected males. Thus, all of our infected and uninfected lines come from the same genetic background. We carried out three further generations of backcrossing of infected females to uninfected males in the lab to effectively remove any residual genetic variation between the two groups. Details of rearing conditions are described in Negri et al. (2006). The natural pattern of sexual reproduction and the additional backcrossing done in the lab ensure the similarity of nuclear genetic backgrounds. We also tested mitochondrial gene sequences in Zyginidia samples from different Italian localities, both infected and uninfected, and they were all nearly identical (Negri I., unpublished data).

Through principal component analysis, we have showed that Wolbachia-induced changes in the host transcriptome are mainly sex-independent, and cannot be explained only by the sex reversal of genetic males. Previous transcriptomic studies on Wolbachia have reported changes in the expression of genes unrelated to the reproductive phenotype. For instance, Wolbachia infection in Armadillidium vulgare triggered the overexpression of immunerelated genes (Chevalier et al., 2012). In the parasitoid wasp Asobara tabida, endosymbiont infection or lack thereof was associated with changes in expression of genes related to female reproductive development, iron and oxidative stress regulation, and immune recognition (Kremer et al., 2009, 2012). Artificial infection of Anopheles cell cultures by Wolbachia, surprisingly caused down-regulation of immune, stress response and detoxification genes (Hughes et al., 2011). Wolbachia-inoculated Drosophila cell lines exhibited differential expression of several GO categories not directly related to reproduction, including antimicrobial humoral response, ion homeostasis, response to unfolded protein and response to chemical stimulus (Xi et al., 2008). In Aedes aegypti, Wolbachia was shown to manipulate the expression of a metalloprotease gene through induction of a specific host miRNA (Hussain et al., 2011). Apart from such direct evidence, the observation of various forms of fitness cost in the feminized males, is consistent with the idea that sex reversal is not the sole effect of feminizing Wolbachia (Moreau et al., 2001; Rigaud and Moreau, 2004). Nevertheless, our study is the first one to quantitatively demonstrate that infection itself has a larger effect than that of sex reversion, through PCA of all of the available gene expression levels.

Lack of replicates meant that we could not quantitatively identify differentially expressed genes between the lines because we could not calculate variances. Instead, we focused on the global patterns of gene expression by applying PCA to gene expression values. Thousands of loci (each acting as one observation point) were used to generate the PCs. Antibiotic treatment and different genetic backgrounds could have been two potential sources of systematic bias in this type of analysis; they could have generated similar clustering patterns and confounded the interpretation of results. However, through the single-population sampling and the repeated backcrossing scheme, we avoided both sources of confusion.

Based on the PCA results, we encourage the use of biochemical bottom-up approaches focusing on the whole Wolbachia effect rather than the specific sex inversion event. Wolbachia's effect is perceivably mediated by molecules secreted into the host cell or expressed on the outer membrane surface of the bacteriumcontaining vesicles. Wolbachia cannot be maintained in cell-free cultures indefinitely; but there are protocols to keep them alive in synthetic media for several hours (Rasgon et al., 2006; Gamston and Rasgon, 2007). In such a setting, the molecules released into the medium can be detected and purified using chromatographic and/or mass spectrometric approaches. Appropriate methods can be used, too, for isolation and characterization of surface molecules from the bacterium-containing vesicles. Pull-down experiments on the host proteins by these Wolbachia released or surface molecules might reveal the initial cellular targets of the endosymbiont-host interaction.

\section{REFERENCES}

Altschul, S. F., Gish, W., Miller, W., Myers, E. W., and Lipman, D. J. (1990). Basic local alignment search tool. J. Mol. Biol. 215, 403-410. doi: 10.1016/S00222836(05)80360-2

Awrahman, Z. A., Champion de Crespigny, F., and Wedell, N. (2014). The impact of Wolbachia, male age and mating history on cytoplasmic incompatibility and sperm transfer in Drosophila simulans. J. Evol. Biol. 27, 1-10. doi: 10.1111/jeb.12270

Ballard, J. W. O., and Melvin, R. G. (2007). Tetracycline treatment influences mitochondrial metabolism and mtDNA density two generations after treatment in Drosophila. Insect Mol. Biol. 16, 799-802. doi: 10.1111/j.1365-2583.2007. 00760.x

Casiraghi, M., McCall, J. W., Simoncini, L., Kramer, L. H., Sacchi, L., Genchi, C., et al. (2002). Tetracycline treatment and sex-ratio distortion: a role for Wolbachia in the moulting of filarial nematodes? Int. J. Parasitol. 32, 1457-1468. doi: 10.1016/S0020-7519(02)00158-3

Chevalier, F., Herbinière-Gaboreau, J., Charif, D., Mitta, G., Gavory, F., Wincker, P., et al. (2012). Feminizing Wolbachia: a transcriptomics approach with insights on the immune response genes in Armadillidium vulgare. BMC Microbiol. 12:S1. doi: 10.1186/1471-2180-12-S1-S1

Cordaux, R., Bouchon, D., and Grève, P. (2011). The impact of endosymbionts on the evolution of host sex-determination mechanisms. Trends Genet. 27, 332-341. doi: 10.1016/j.tig.2011.05.002

Cordaux, R., Michel-Salzat, A., and Bouchon, D. (2001). Wolbachia infection in crustaceans: novel hosts and potential routes for horizontal transmission. J. Evol. Biol. 14, 237-243. doi: 10.1046/j.1420-9101.2001.00279.x 
Darby, A. C., Armstrong, S. D., Bah, G. S., Kaur, G., Hughes, M. A., Kay, S. M., et al. (2012). Analysis of gene expression from the Wolbachia genome of a filarial nematode supports both metabolic and defensive roles within the symbiosis. Genome Res. 22, 2467-2477. doi: 10.1101/gr.138420.112

Dunn, A. M., Andrews, T., Ingrey, H., Riley, J., and Wedell, N. (2006). Strategic sperm allocation under parasitic sex-ratio distortion. Biol. Lett. 2, 78-80. doi: 10.1098/rsbl.2005.0402

Ewing, B., and Green, P. (1998). Base-calling of automated sequencer traces using phred. II. Error probabilities. Genome Res. 8, 186-194. doi: 10.1101/gr 8.3.175

Fischer, K., Beatty, W. L., Jiang, D., Weil, G. J., and Fischer, P. U. (2011). Tissue and stage-specific distribution of Wolbachia in Brugia malayi. PLoS Negl. Trop. Dis. 5:e1174. doi: 10.1371/journal.pntd.0001174

Foster, J., Ganatra, M., Kamal, I., Ware, J., Makarova, K., Ivanova, N., et al. (2005). The Wolbachia genome of Brugia malayi: endosymbiont evolution within a human pathogenic nematode. PLoS Biol. 3:e121. doi: 10.1371/journal.pbio.0030121

Fridmann-Sirkis, Y., Stern, S., Elgart, M., Galili, M., Zeisel, A., Shental, N., et al. (2014). Delayed development induced by toxicity to the host can be inherited by a bacterial-dependent, transgenerational effect. Front. Genet. 5:27. doi: 10.3389/fgene.2014.00027

Gamston, C., and Rasgon, J. (2007). Maintaining Wolbachia in cell-free medium. J. Vis. Exp. 5:223. doi: 10.3791/223

Gempe, T., and Beye, M. (2011). Function and evolution of sex determination mechanisms, genes and pathways in insects. Bioessays 33, 52-60. doi: 10.1002/ bies. 201000043

Gottlieb, Y., Zchori-Fein, E., Werren, J. H., and Karr, T. L. (2002). Diploidy restoration in Wolbachia-infected Muscidifurax uniraptor (Hymenoptera: Pteromalidae). J. Invertebr. Pathol. 81, 166-174. doi: 10.1016/S0022-2011(02) 00149-0

Götz, S., García-Gómez, J. M., Terol, J., Williams, T. D., Nagaraj, S. H., Nueda, M. J., et al. (2008). High-throughput functional annotation and data mining with the Blast2GO suite. Nucleic Acids Res. 36, 3420-3435. doi: 10.1093/nar/gkn176

Hoffmann, A. A., Turelli, M., and Harshman, L. G. (1990). Factors affecting the distribution of cytoplasmic incompatibility in Drosophila simulans. Genetics 126, 933-948.

Hughes, G. L., Ren, X., Ramirez, J. L., Sakamoto, J. M., Bailey, J. A., Jedlicka, A. E., et al. (2011). Wolbachia infections in Anopheles gambiae cells: transcriptomic characterization of a novel host-symbiont interaction. PLoS Pathog. 7:e1001296. doi: 10.1371/journal.ppat.1001296

Hussain, M., Frentiu, F. D., Moreira, L. A., O’Neill, S. L., and Asgari, S. (2011) Wolbachia uses host microRNAs to manipulate host gene expression and facilitate colonization of the dengue vector Aedes aegypti. Proc. Natl. Acad. Sci. U.S.A. 108, 9250-9255. doi: 10.1073/pnas.1105469108

Iturbe-Ormaetxe, I., Burke, G. R., Riegler, M., and Neill, S. L. O. (2005). Distribution, expression, and motif variability of ankyrin domain genes in Wolbachia pipientis. J. Bacteriol., 187, 5136-5145. doi: 10.1128/JB.187.15.5136

Jaenike, J. (2009). Coupled population dynamics of endosymbionts within and between hosts. Oikos 118, 353-362. doi: 10.1111/j.1600-0706.2008 17110.x

Klasson, L., Walker, T., Sebaihia, M., Sanders, M. J., Quail, M. A., Lord, A., et al. (2008). Genome evolution of Wolbachia strain wPip from the Culex pipiens group. Mol. Biol. Evol. 25, 1877-1887. doi: 10.1093/molbev/msn133

Kremer, N., Charif, D., Henri, H., Gavory, F., Wincker, P., Mavingui, P., et al. (2012) Influence of Wolbachia on host gene expression in an obligatory symbiosis. BMC Microbiol. 12:S7. doi: 10.1186/1471-2180-12-S1-S7

Kremer, N., Voronin, D., Charif, D., Mavingui, P., Mollereau, B., and Vavre, F. (2009). Wolbachia interferes with ferritin expression and iron metabolism in insects. PLoS Pathog. 5:e1000630. doi: 10.1371/journal.ppat.1000630

Li, H., and Durbin, R. (2009). Fast and accurate short read alignment with Burrows-Wheeler transform. Bioinformatics 25, 1754-1760. doi: 10.1093/bioinformatics/btp324

Liu, C., Wang, J. L., Zheng, Y., Xiong, E. J., Li, J. J., Yuan, L. L., et al. (2014). Wolbachia-induced paternal defect in Drosophila is likely by interaction with the juvenile hormone pathway. Insect Biochem. Mol. Biol. 49, 49-58. doi: 10.1016/j. ibmb.2014.03.014

Ma, W. J., Vavre, F., and Beukeboom, L. W. (2014). Manipulation of arthropod sex determination by endosymbionts: diversity and molecular mechanisms. Sex. Dev. 8, 59-73. doi: 10.1159/000357024
Milani, L., Ghiselli, F., Maurizii, M. G., and Passamonti, M. (2011). Doubly uniparental inheritance of mitochondria as a model system for studying germ line formation. PLoS ONE 6:e28194. doi: 10.1371/journal.pone.0028194

Moreau, J., Bertin, A., Caubet, Y., and Rigaud, T. (2001). Sexual selection in an isopod with Wolbachia-induced sex reversal: males prefer real females. J. Evol. Biol. 14, 388-394. doi: 10.1046/j.1420-9101.2001.00292.x

Mortazavi, A., Williams, B. A., McCue, K., Schaeffer, L., and Wold, B. (2008) Mapping and quantifying mammalian transcriptomes by RNA-Seq. Nat. Methods 5, 621-628. doi: 10.1038/NMETH.1226

Negri, I. (2012). Wolbachia as an "infectious" extrinsic factor manipulating host signaling pathways. Front. Endocrinol. (Lausanne) 2:115. doi: 10.3389/fendo.2011. 00115

Negri, I., Franchini, A., Gonella, E., Daffonchio, D., Mazzoglio, P. J., Mandrioli, M. et al. (2009a). Unravelling the Wolbachia evolutionary role: the reprogramming of the host genomic imprinting. Proc. R. Soc. B Biol. Sci. 276, 2485-2491. doi: 10.1098/rspb.2009.0324

Negri, I., Franchini, A., Mandrioli, M., Mazzoglio, P. J., and Alma, A. (2008) The gonads of Zyginidia pullula males feminized by Wolbachia pipientis. Bull. Insectol. 61, 213-214.

Negri, I., Mazzoglio, P. J., Franchini, A., Mandrioli, M., and Alma, A. (2009b). Male or female? The epigenetic conflict between a feminizing bacterium and its insect host. Commun. Integr. Biol. 2, 1-2. doi: 10.4161/cib.2.6.9559

Negri, I., Pellecchia, M., Grève, P., Daffonchio, D., Bandi, C., and Alma, A. (2010). Sex and stripping: the key to the intimate relationship between Wolbachia and host? Commun. Integr. Biol. 3, 1-6. doi: 10.4161/cib.3.2.10520

Negri, I., Pellecchia, M., Mazzoglio, P. J., Patetta, A., and Alma, A. (2006) Feminizing Wolbachia in Zyginidia pullula (Insecta, Hemiptera), a leafhopper with an XX/X0 sex-determination system. Proc. R. Soc. B Biol. Sci. 273, 2409-2416. doi: 10.1098/rspb.2006.3592

Noda, H., Miyoshi, T., and Koizumi, Y. (2002). In vitro cultivation of Wolbachia in insect and mammalian cell lines. In Vitro Cell. Dev. Biol. Anim. 38, 423-427. doi: 10.1290/1071-2690(2002)038\%3C0423:IVCOWI\%3E2.0.CO;2

Pannebakker, B. A., Pijnacker, L. P., Zwaan, B. J., and Beukeboom, L. W. (2004). Cytology of Wolbachia-induced parthenogenesis in Leptopilina clavipes (Hymenoptera: Figitidae). Genome 47, 299-303. doi: 10.1139/g03-137

Rasgon, J. L., Gamston, C. E., and Ren, X. (2006). Survival of Wolbachia pipientis in cell-free medium. Appl. Environ. Microbiol. 72, 6934-6937. doi: 10.1128/AEM. 01673-06

R Development Core Team. (2011). R: a Language and Environment for Statistical Computing (Vienna: R Foundation for Statistical Computing). Available online at: http://www.R-project.org

Rigaud, T., and Moreau, J. (2004). A cost of Wolbachia-induced sex reversal and female-biased sex ratios: decrease in female fertility after sperm depletion in a terrestrial isopod. Proc. R. Soc. B Biol. Sci. 271, 1941-1946. doi: 10.1098/rspb. 2004.2804

Riparbelli, M. G., Giordano, R., Ueyama, M., and Callaini, G. (2012). Wolbachiamediated male killing is associated with defective chromatin remodeling. PLoS ONE 7:e30045. doi: 10.1371/journal.pone.0030045

Robinson, M. D., McCarthy, D. J., and Smyth, G. K. (2010). edgeR: a Bioconductor package for differential expression analysis of digital gene expression data. Bioinformatics 26, 139-140. doi: 10.1093/bioinformatics/btp616

Robinson, M. D., and Oshlack, A. (2010). A scaling normalization method for differential expression analysis of RNA-seq data. Genome Biol. 11:R25. doi: 10.1186/gb-2010-11-3-r25

Sánchez, L. (2008). Sex-determining mechanisms in insects. Int. J. Dev. Biol. 52, 837-856. doi: 10.1387/ijdb.072396ls

Schulenburg, J. H., Hurst, G. D., Huigens, T. M., van Meer, M. M., Jiggins, F. M., and Majerus, M. E. (2000). Molecular evolution and phylogenetic utility of Wolbachia ftsZ and wsp gene sequences with special reference to the origin of male-killing. Mol. Biol. Evol. 17, 584-600. doi: 10.1093/oxfordjournals.molbev.a026338

Serbus, L. R., Casper-Lindley, C., Landmann, F., and Sullivan, W. (2008). The genetics and cell biology of Wolbachia-host interactions. Annu. Rev. Genet. 42, 683-707. doi: 10.1146/annurev.genet.41.110306.130354

Sugimoto, T. N., and Ishikawa, Y. (2012). A male-killing Wolbachia carries a feminizing factor and is associated with degradation of the sex-determining system of its host. Biol. Lett. 8, 412-415. doi: 10.1098/rsbl.2011.1114

Sze, S.-H., Dunham, J. P., Carey, B., Chang, P. L., Li, F., Edman, R. M., et al. (2012). A de novo transcriptome assembly of Lucilia sericata (Diptera: Calliphoridae) 
with predicted alternative splices, single nucleotide polymorphisms and transcript expression estimates. Insect Mol. Biol. 21, 205-221. doi: 10.1111/j.13652583.2011.01127.x

Taylor, D. R. (1990). Evolutionary consequences of cytoplasmic sex ratio distorters. Evol. Ecol. 4, 235-248. doi: 10.1007/BF02214332

The International Aphid Genomics Consortium. (2010). Genome sequence of the pea aphid Acyrthosiphon pisum. PLoS Biol. 8:e1000313. doi: 10.1371/journal. pbio. 1000313

Turelli, M. (1994). Evolution of incompatibility-inducing microbes and their hosts. Evolution 48, 1500-1513. doi: 10.2307/2410244

Verhulst, E. C., van de Zande, L., and Beukeboom, L. W. (2010). Insect sex determination: it all evolves around transformer. Curr. Opin. Genet. Dev. 20, 376-383. doi: 10.1016/j.gde.2010.05.001

Weeks, A. R., and Breeuwer, J. A. J. (2001). Wolbachia-induced parthenogenesis in a genus of phytophagous mites. Proc. R. Soc. B Biol. Sci. 268, 2245-2251. doi: 10.1098/rspb.2001.1797

Werren, J. H., Baldo, L., and Clark, M. E. (2008). Wolbachia: master manipulators of invertebrate biology. Nat. Rev. Microbiol. 6, 741-751. doi: 10.1038/ nrmicro1969

Werren, J. H., Zhang, W., and Guo, L. R. (1995). Evolution and phylogeny of Wolbachia: reproductive parasites of arthropods. Proc. R. Soc. B Biol. Sci. 261, 55-63. doi: 10.1098/rspb.1995.0117

White, J. A., Giorgini, M., Strand, M. R., and Pennacchio, F. (2013). "Arthropod endosymbiosis and evolution," in Arthropod Biology and Evolution, eds A. Minelli, G. Boxshall, and G. Fusco (Berlin; Heidelberg: Springer-Verlag), 441-477.

Woolfit, M., Iturbe-Ormaetxe, I., McGraw, E. A., and O’Neill, S. L. (2009). An ancient horizontal gene transfer between mosquito and the endosymbiotic bacterium Wolbachia pipientis. Mol. Biol. Evol. 26, 367-374. doi: 10.1093/molbev/msn253

Wu, M., Sun, L. V., Vamathevan, J., Riegler, M., Deboy, R., Brownlie, J. C., et al. (2004). Phylogenomics of the reproductive parasite Wolbachia pipientis wMel: a streamlined genome overrun by mobile genetic elements. PLoS Biol. 2:E69. doi: 10.1371/journal.pbio.0020069

Xi, Z., Gavotte, L., Xie, Y., and Dobson, S. L. (2008). Genome-wide analysis of the interaction between the endosymbiotic bacterium Wolbachia and its Drosophila host. BMC Genomics 9:1. doi: 10.1186/1471-2164-9-1

Ye, J., Fang, L., Zheng, H., Zhang, Y., Chen, J., Zhang, Z., et al. (2006). WEGO: a web tool for plotting GO annotations. Nucleic Acids Res. 34, W293-W297. doi: 10.1093/nar/gkl031

Zeh, J. A., Bonilla, M. M., Adrian, A. J., Mesfin, S., and Zeh, D. W. (2012). From father to son: transgenerational effect of tetracycline on sperm viability. Sci. Rep. 2:375. doi: $10.1038 /$ srep00375

Zerbino, D. R., and Birney, E. (2008). Velvet: algorithms for de novo short read assembly using de Bruijn graphs. Genome Res. 18, 821-829. doi: 10.1101/gr. 074492.107

Conflict of Interest Statement: The authors declare that the research was conducted in the absence of any commercial or financial relationships that could be construed as a potential conflict of interest.

Received: 30 April 2014; accepted: 30 July 2014; published online: 01 September 2014 Citation: Asgharian H, Chang PL, Mazzoglio PJ and Negri I (2014) Wolbachia is not all about sex: male-feminizing Wolbachia alters the leafhopper Zyginidia pullula transcriptome in a mainly sex-independent manner. Front. Microbiol. 5:430. doi: 10.3389/ fmicb.2014.00430

This article was submitted to Microbial Symbioses, a section of the journal Frontiers in Microbiology.

Copyright () 2014 Asgharian, Chang, Mazzoglio and Negri. This is an open-access article distributed under the terms of the Creative Commons Attribution License (CC BY). The use, distribution or reproduction in other forums is permitted, provided the original author(s) or licensor are credited and that the original publication in this journal is cited, in accordance with accepted academic practice. No use, distribution or reproduction is permitted which does not comply with these terms. 